\title{
Pays et intercommunalité, quelles conséquences de la réforme des collectivités territoriales?
}

\section{Denis Lepicier, Gwénaël Doré and Abdoul Diallo}

\section{(2) OpenEdition}

Electronic version

URL: http://journals.openedition.org/economierurale/4521

DOI: 10.4000/economierurale.4521

ISSN: 2105-2581

\section{Publisher}

Société Française d'Économie Rurale (SFER)

\section{Printed version}

Date of publication: 20 December 2014

Number of pages: $61-74$

ISSN: 0013-0559

\section{Electronic reference}

Denis Lepicier, Gwénaël Doré et Abdoul Diallo, « Pays et intercommunalité, quelles conséquences de la réforme des collectivités territoriales? 》, Économie rurale [En ligne], 344 | novembre-décembre 2014, mis en ligne le 01 janvier 2016, consulté le 20 avril 2019. URL : http://journals.openedition.org/ economierurale/4521; DOI : 10.4000/economierurale.4521 


\title{
FAITS ET CHIFFRES
}

\section{Pays et intercommunalité, quelles conséquences de la réforme des collectivités territoriales?}

\author{
Denis LEPICIER • UMR 1041 CESAER, INRA, AgroSup Dijon \\ Gwénaël DORE • Institut National du Développement Local (INDL); \\ UMR 1048 SAD-APT, INRA, AgroParistech \\ Abdoul DIALLO • UMR 1041 CESAER, INRA, AgroSup Dijon
}

Cet article analyse les conséquences de la loi de réforme des collectivités territoriales du 16 décembre 2010 sur l'organisation des territoires français. En partant d'une caractérisation spatiale et socioéconomique de Pays et de leur structuration intercommunale, le texte examine les perspectives d'articulation spatiale entre Pays et communautés qui ressortent des schémas départementaux de coopération intercommunale adoptés en 2011-2012. L'article conclut sur le constat d'une perspective de restructuration significative de l'intercommunalité, mais qui remet peu en cause la pertinence d'une échelle d'action publique infra-régionale et supra-communautaire.

Comme dans la plupart des Pays occidentaux, les années 1980 ont marqué le point de départ en France de nombreuses réformes de l'action publique combinant décentralisation de l'organisation de l'État et territorialisation des politiques publiques. Le processus de décentralisation, marqué d'abord par les lois Deferre de 1982 instituant les collectivités régionales, s'est poursuivi à l'échelle locale dans le cadre de la loi de l'Administration Territoriale de la République de 1992 en créant les Établissements publics de coopération intercommunale (EPCI) à fiscalité propre. Le processus de territorialisation, entendu comme une approche des politiques publiques qui prend en compte les spécificités des territoires infra-régionaux dans les processus de développement, est expérimenté en France dans les années 1970-1980 à travers les plans d'aménagement ruraux puis les chartes intercommunales. Il est ensuite impulsé par les projets européens Leader, avant de trouver une première reconnaissance dans la loi Pasqua (Loi d'orientation pour l'aménagement et le développement du territoire - LOADT) du 4 février 1995 qui institue les Pays comme une forme souple de coopération intercommunale, qui n'a pas le statut de collectivité territoriale, constituée à une échelle supra-communautaire et jugée plus pertinente pour engager des stratégies de développement territorial. La loi d'orientation pour l'aménagement et le développement durable du territoire (LOADDT), dite loi Voynet du 25 juin 1999 renforce l'articulation entre les Pays et les EPCI et incite à la coopération accrue entre territoires ruraux et urbains ( $c f$. encadré 1). Pour le législateur, les Pays devaient reposer ainsi sur une organisation en communautés de communes et/ou d'agglomération, et implicitement constituer un espace de coopération d'EPCI à fiscalité propre.

$\mathrm{Au}$ cours des années 2000, ces deux formes de structuration territoriale se sont généralisées. Du côté de l'intercommunalité, les lois du 12 juillet 1999 et du 13 août $2004^{1}$, en complétant et en clarifiant son fonctionnement, vont accélérer son développement en encourageant la logique de

1. Loi relative aux libertés et responsabilités locales. 
projet et la mutualisation des ressources fiscales par la fiscalité unique. La loi de Réforme des collectivités territoriales (RCT) du 16 décembre 2010 affiche l'objectif d'atteindre la couverture exhaustive du territoire en intercommunalités pour le $1^{\text {er }}$ janvier 2014. Pour cela, elle accorde un rôle accru aux préfets de département dans l'élaboration et l'application des Schémas départementaux de coopération intercommunale (SDCI) qui incite à l'extension ou à la fusion des EPCI à fiscalité propre et ceux de type syndical (SIVU, SIVOM, syndicat mixte $)^{2}$ existants en fixant quelques critères de rationalisation de l'intercommunalité 3 . Il s'agit de corriger un grief souvent porté au processus de développement de l'intercommunalité, qui est celui de la taille trop limitée d'une partie des groupements créés depuis les années 2000, notamment les communautés de communes rurales souvent constituées à l'échelle cantonale. Il laisse dans les faits une large marge d'appréciation de la taille pertinente et des critères de cohérence territoriale aux préfets et aux élus locaux. Le projet d'abolition des provinces, échelon intermédiaire entre municipalités et régions, en Italie, ou le processus de fusion de communes conduit en Grèce en 2011 montrent que la question relative à la décentralisation est une question d'actualité en Europe.

Dans le même temps, les Pays se sont généralisés en France comme forme de coopération entre plusieurs EPCI et dans de nombreux cas entre communautés de communes et d'agglomérations dans la perspective

2. Un peu plus de 15000 syndicats intercommunaux existent en 2011 au côté des EPCI à fiscalité propre, dont plus de 10000 sont spécialisés sur la gestion d'un seul service.

3. Les EPCI devront, dans la mesure du possible, regrouper au moins 5000 habitants et viser à améliorer « la cohérence spatiale des établissements publics de coopération intercommunale à fiscalité propre au regard notamment du périmètre des unités urbaines au sens de l'INSEE et des schémas de cohérence territoriale » (art. 16 de la loi RCT). visée par le législateur d'articuler espaces ruraux et espaces urbains. L'échelle des Pays est devenue un niveau de contractualisation privilégié pour les conseils régionaux (Doré, 2014) ${ }^{4}$, trouvant ainsi une manière efficace de contourner les effets dispersifs d'un grand nombre de communautés ${ }^{5}$. Toutefois, des critiques récurrentes ont été portées à l'encontre des Pays qui représenteraient une strate supplémentaire malgré les constats des effets positifs des Pays en faveur de la coopération intercommunale (rapport Balladur, 2009) ${ }^{6}$, d'où l'abrogation de l'article 22 de la loi relatif aux Pays par la loi RCT mettant fin à la reconnaissance par l'État de nouveaux Pays. Plusieurs régions ont d'ores et déjà affirmé qu'elles continueront à mobiliser cette échelle intercommunautaire pour mettre en œuvre leur politique territoriale. Enfin, la loi de modernisation de l'action publique territoriale et d'affirmation des métropoles (MAPAM) du 27 janvier 2014 crée les pôles d'équilibre territoriaux et ruraux (PETR), donnant ainsi un nouveau prolongement législatif aux Pays.

Les conséquences des différents volets de la réforme des collectivités territoriales sur la structuration territoriale de l'action publique, tant du point de vue de l'articulation des échelles de l'action publique régionale et locale que du point de vue de la répartition des compétences entre ces niveaux, méritent attention. Le présent article se focalise sur l'analyse de l'évolution des périmètres des

4. Doré G. (2014). La contractualisation infra-régionale des régions françaises, Revue d'économie régionale et urbaine, $\mathrm{n}^{\circ} 1$, p. 157-172.

5. Cet échelon d'action publique complémentaire à ceux des collectivités locales est plus original à l'échelle européenne. En Allemagne, par exemple, des coopérations intercommunales existent au-delà des périmètres des districts (par exemple pour la gestion de projets européens Leader), mais constituent des formes moins institutionnalisées.

6. Balladur E. (2009). Comité pour la réforme des collectivités locales - Il est temps de déciderRapport au président de la République. Paris, La Documentation française, Fayard, 174 p. 


\section{Encadré 1. Pays et intercommunalités, des acteurs complémentaires du développement local}

Les communautés sont des Établissements publics de coopération intercommunale (EPCI) à fiscalité propre, c'est-à-dire des regroupements de communes reposant sur une mise en commun de compétences et des ressources fiscales. En 2014, quatre types de communautés correspondent à une gradation de la taille de population et de l'intégration de compétences : les communautés de communes, les communautés d'agglomération (à partir de 50000 habitants), les communautés urbaines (à partir de 250000 habitants) et les métropoles à partir de 400000 habitants. Les EPCI interviennent dans les domaines de compétences qui leur sont transférées par les communes, parmi lesquelles le développement économique et de l'aménagement de l'espace sont obligatoires. Ceci est ou peut être complété par une large palette d'autres compétences en matière de développement touristique, d'environnement, de logement et d'habitat, de politique de la ville ou encore d'équipements culturels ou sportifs et d'action sociale.

Les Pays regroupent quant à eux plusieurs communautés autour de projets de développement, selon une formule souple de coopération, et ils sont constitués sous diverses formes juridiques : syndicat (reposant sur des contributions financières des membres et non sur des ressources fiscales perçues par le syndicat), groupement d'intérêt public (entité regroupant des structures publiques et parfois privées), association, voire simple convention entre communautés. Les Pays se centrent sur des missions de planification stratégique du développement du territoire dans une perspective globale et transversale (charte de territoire) et d'animation du territoire en vue de la mise en œuvre d'un plan d'action (contrat de Pays) par les acteurs économiques, associatifs et publics locaux.
EPCI proposée dans les SDCI élaborés en 2011 et 2012 et de leur cohérence avec les périmètres des Pays actuellement reconnus. Sur le plan théorique, ce questionnement relève de l'économie régionale et de l'économie publique. Sur le premier plan, il s'agit d'appréhender les dynamiques économiques de ces périmètres d'action publique à partir des formes de polarisation (de population et d'emplois) des territoires. Le second plan renvoie notamment aux travaux de fédéralisme fiscal s'interrogeant sur l'architecture administrative optimale (nombre de niveaux et géographie du découpage territorial à chacun des niveaux).

En partant d'une actualisation de la caractérisation spatiale et socio-économique de Pays (Aubert et al., 2006) ${ }^{7}$, de leur

7. Aubert F., Lépicier D., Perrier-Cornet P. (2006). Structure économique des territoires : une analyse des disparités micro-régionales à l'échelle des Pays en France. Revue d'économie régionale et urbaine, $\mathrm{n}^{\circ} 2$, p. 203-225. fonctionnement (Doré, 2013) ${ }^{8}$ et de leur structuration intercommunale en 2010, antérieure à la réforme (section 1), nous examinons les perspectives d'articulation des périmètres des Pays avec la nouvelle carte des EPCI qui ressort des SDCI récemment adoptés dans les départements français (section 2). Sont enfin examinées les conséquences de ces évolutions sur le devenir des territoires de projet particulièrement impactés (section 3).

L'absence d'avancées majeures de la RCT sur les critères de rationalisation de l'intercommunalité en termes de taille critique et d'engagement dans une

8. Doré G. (2013). Les territoires de projets entre intégration institutionnelle et démarches de développement durable, In Carrière J.-P., Demazière C., Petrea R., Filimon L. (dir.), La mise en cuvre du développement territorial durable : déclinaisons franco-roumaines, University of Oradea (Romania) \& IPA-PE/CITERES, Université de Tours, Paris, L'Harmattan. 


\section{Encadré 2. Méthodologie et sources de données}

La typologie spatiale des 400 Pays $^{1}$ (dont la composition communale au $1^{\text {er }}$ janvier 2011 provient de la DATAR) reprend les mêmes principes que celle de 2006 (Aubert et al., 2006) en les ajustant à la refonte du ZAU 2010 de l'INSEE. Elle distingue 10 types spatiaux regroupés en 4 grands types (Pays d'agglomération et urbains, périurbains, ruraux et composites) selon 2 critères : i) part des populations communales agrégées du Pays, localisée dans chaque classe du ZAU en 2010, ii) nombre de grands, moyens et petits pôles dans le Pays d'après le ZAU 2010 (cf. Annexe). La composition communale et

1. Dont 25 contrats de développement durables de Rhône-Alpes (CDDRA) non officiellement labellisés Pays. Nous les englobons sous le vocable de Pays dans le reste de l'article. le régime fiscal des EPCI existants en 2010 proviennent de la Direction générale des collectivités locales.

L'évolution de la carte intercommunale (section 2) est traitée à partir de l'analyse de 63 SDCI sur les 73 adoptés en 2011-2012². Les périmètres intercommunaux retenus recoupent ceux de 294 Pays sur les 400 existants au $1^{\text {er }}$ janvier 2013. L'analyse des SDCI a permis de constituer une base de données de l'évolution de la carte intercommunale avant/après réforme et de comparer ces évolutions à la carte des Pays.

2. N'ont pas été traités les SDCI des départements d'Île-de-France non concernés par les Pays et ceux de quelques autres départements dans lesquels l'information n'était pas disponible ou exploitable. logique de projet de territoire plus ambitieuse, nous conduit à faire l'hypothèse d'une «persistance » de l'échelle de Pays comme échelle de coopération d'intercommunalités articulant les villes, les espaces périurbains et les bassins de vie plus ruraux.

\section{Des configurations territoriales et institutionnelles variées des Pays}

La finalité de la constitution d'un Pays étant l'élaboration d'une stratégie intercommunautaire de développement territorial, on s'attend à ce que les caractéristiques spatiales et socio-économiques conditionnent la dimension et la cohérence des périmètres constitués. Toutefois, les Pays sont des territoires fondés sur l'adhésion volontaire des communes et sont construits sur un registre essentiellement politique (Aubert et al., 2006). Il en résulte des formes très variées de configurations territoriales de Pays.

\section{Les Pays, des territoires de plus en plus urbains}

Aubert et al. (2006) proposaient une typologie de la structuration spatiale des Pays (existants au $1^{\text {er }}$ janvier 2004) basée sur 2 principaux critères : d'une part, la répartition de la population du Pays selon les catégories d'espace définies dans le Zonage en aire urbaine et de l'espace rural (Zauer, 2002) de l'INSEE', et d'autre part le degré de polarisation caractérisé par le nombre de villes ou bourgs ruraux tels que définis par ce même zonage. Quatre grands types spatiaux de Pays étaient ainsi proposés : les Pays urbains, périurbains, ruraux et composites. Une actualisation de cette typologie est ici proposée prenant en compte la mise à jour du zonage

9. Selon le zonage en aires urbaines de 2002 de l'INSEE, l'espace à dominante urbaine regroupe les pôles urbains, c'est-à-dire les unités urbaines de plus de 5000 emplois et les communes périurbaines, définies comme les communes dont plus de $40 \%$ des actifs ayant un emploi travail dans le pôle urbain. L'espace à dominante rurale regroupe le reste du territoire. 
en aires urbaines (ZAU, INSEE 2011) $)^{10}$ et la géographie des territoires de projets ( $c f$. encadré 2) au $1^{\text {er }}$ janvier 2011. Cette nouvelle typologie conduit à observer un plus grand nombre de territoires structurés au sein des aires urbaines, soit plus d'un Pays sur deux alors qu'il en représentait qu'un sur trois en 2004, une nette augmentation du nombre de territoires composites et une très forte diminution des territoires ruraux (au sens de hors aire urbaine) dont la proportion passe de plus de quatre Pays sur dix en 2004 à moins d'un sur dix en 2011.

Si les Pays ont été longtemps associés aux territoires ruraux, ce n'est plus le cas aujourd'hui. Deux phénomènes complémentaires peuvent l'expliquer. On observe tout d'abord, une extension progressive de la couverture des Pays au cours des années 2000 : 109 nouveaux Pays ont été créés entre 2004 et 2011 parmi lesquels près de $70 \%$ sont des Pays urbains. Ensuite, l'évolution de la géographie de la localisation de la population et des pratiques de mobilité domiciletravail (dont le zonage en aires urbaines de 2010 rend compte) révèle une accentuation nette de l'influence des métropoles, agglomérations et villes moyennes. Ainsi, $82 \%$ des 356 grands et moyens pôles urbains appartiennent à un Pays. La forte extension du nombre de communes périurbaines mise en évidence par le nouveau zonage fait évoluer un grand nombre de Pays ruraux vers une configuration spatiale composite mixant villes moyennes, petites villes, communes périurbaines et communes rurales. Les pôles ruraux (petits pôles) sont encore plus systématiquement structurants des Pays (92\% d'entre eux sont dans des Pays), mais le sont le plus souvent sous la forme d'un maillage de petites villes de Pays ruraux ou de petites villes avec une ville moyenne dans le cas des Pays composites.

10. INSEE (2011). Le nouveau zonage en aires urbaines de 2010. Poursuite de la périurbanisation et croissance des grandes aires urbaines, Insee Première, $\mathrm{n}^{\circ}$ 1375, octobre 2011.
Les Pays d'agglomération et urbains représentent donc aujourd'hui la configuration spatiale de Pays la plus fréquente (38\% des Pays) qui regroupe $60 \%$ de la population totale appartenant à un Pays et plus de 130000 habitants en moyenne ( $c f$. tableau 1). $40 \%$ d'entre eux concernent des agglomérations de plus de 50000 habitants (Brest, Caen, Amiens, Le Havre, Le Mans ou Rennes) et leur couronne périurbaine, mais rarement au-delà, alors que les Pays urbains sont structurés autour de villes moyennes (Aurillac, Beaune, Digne, Guingamp, Sarrebourg ou encore Soissons), de leur couronne périurbaine et débordant parfois sur l'arrière-Pays rural. Ces derniers connaissent une forte dynamique démographique du fait d'une attractivité migratoire supérieure aux Pays d'agglomération.

Les Pays périurbains sont quant à eux de dimension beaucoup plus réduite en nombre d'habitants du fait de la plus faible densité de population de ces espaces par rapport au précédent et du plus faible nombre de communes regroupées. Ces Pays occupent une position spatiale excentrée au sein des aires urbaines, débordant souvent sur l'espace à dominante rurale le plus proche. Leur organisation en « marguerite » autour des aires urbaines relève d'une logique plutôt défensive (Aubert et al., 2006), profitant de leur forte dynamique démographique nettement supérieure à l'ensemble des autres types.

La structuration en Pays de l'espace rural est plus ancienne, car la politique de Pays impulsée par la loi Pasqua visait plus spécifiquement ces espaces. Cette configuration spatiale caractérisée par le regroupement de communes rurales hors de l'influence de pôles moyens et grands pôles urbains s'est fortement raréfiée. Les Pays ruraux sont logiquement de faible dimension, mais relativement vastes en nombre de communes. L'ambition de la loi Voynet de renforcer l'articulation ville-campagne 
Tableau 1. Typologie spatiale actualisée des Pays

\begin{tabular}{|c|c|c|c|c|c|c|c|c|}
\hline \multirow{3}{*}{$\begin{array}{l}\text { Typologie spatiale des Pays } \\
\text { Pays d'agglomération et urbains }\end{array}$} & \multirow{2}{*}{\multicolumn{2}{|c|}{ Nb Pays }} & \multicolumn{4}{|c|}{ Distribution nationale } & \multicolumn{2}{|c|}{$\begin{array}{l}\text { Structure moy. } \\
\text { des Pays }\end{array}$} \\
\hline & & & \multicolumn{2}{|c|}{ des communes } & \multicolumn{2}{|c|}{$\begin{array}{l}\text { de la popu- } \\
\text { lation 2009 } \\
(\times 1000)\end{array}$} & $\mathrm{Nb}$ com & $\begin{array}{c}\text { Pop } \\
2009 \\
(x 1000)\end{array}$ \\
\hline & 154 & 38,5 & 12355 & 39,6 & 20465 & 60,0 & 80 & 133 \\
\hline -dont Pays d'agglomération & 62 & 15,5 & 5533 & 17,8 & 13235 & 38,8 & 89 & 213 \\
\hline -dont Pays urbains & 92 & 23,0 & 6822 & 21,9 & 7230 & 21,2 & 74 & 79 \\
\hline Pays périurbains, dont & 62 & 15,5 & 3527 & 11,3 & 3467 & 10,2 & 57 & 56 \\
\hline -Pays périurbains sans relais & 41 & 10,3 & 2060 & 6,6 & 2103 & 6,2 & 50 & 51 \\
\hline -Pays périurbains avec relais & 21 & 5,3 & 1467 & 4,7 & 1364 & 4,0 & 70 & 65 \\
\hline Pays ruraux, dont & 39 & 9,8 & 2644 & 8,5 & 1181 & 3,5 & 68 & 30 \\
\hline -Pays ruraux & 7 & 1,8 & 451 & 1,5 & 152 & 0,4 & 64 & 22 \\
\hline -Pays ruraux de petites villes & 16 & 4,0 & 923 & 3,0 & 384 & 1,1 & 58 & 24 \\
\hline -Pays ruraux maillés & 16 & 4,0 & 1270 & 4,1 & 645 & 1,9 & 79 & 40 \\
\hline Pays composites dont & 145 & 36,3 & 12638 & 40,6 & 8969 & 26,3 & 87 & 62 \\
\hline -Pays composites non polarisés & 55 & 13,8 & 3862 & 12,4 & 1999 & 5,9 & 70 & 36 \\
\hline -Pays composites monopolaires & 55 & 9,8 & 3394 & 10,9 & 2045 & 6,0 & 62 & 37 \\
\hline -Pays composites multipolaires & 51 & 12,8 & 5382 & 17,3 & 4925 & 14,5 & 106 & 97 \\
\hline Ensemble Pays & 400 & 100 & 31164 & 100 & 34081 & 100 & 78 & 85 \\
\hline
\end{tabular}

Source : DATAR, INSEE 2009.

a contribué à la structuration d'une grande majorité des Pays ruraux autour d'un petit pôle ${ }^{11}$ ou de plusieurs (Pays ruraux maillés) - les Pays ruraux ne comptant aucun pôle dans leur périmètre sont de l'ordre de l'exception (7 recensés sur les 400).

Les Pays composites dépassent plus significativement le clivage urbain/rural en regroupant des espaces urbains et ruraux dans de larges périmètres (ce sont ces Pays composites qui regroupent le plus grand nombre de communes en moyenne) favorisant le développement de complémentarités entre et avec les villes moyennes. Cette configuration, permettant aux espaces ruraux de bénéficier potentiellement d'effets d'agglomération, peut apparaître comme la plus proche de l'esprit de la LOADDT.

11. Unité urbaine regroupant de 1500 à 5000 emplois.

\section{Une structuration intercommunale} des Pays avant la réforme de type intercommunautaire

En 2010, la structuration intercommunale des Pays est très forte (96 \% de la population et des communes). Seuls 18 Pays ont une couverture intercommunale inférieure à $80 \%$ (en population). Les 400 territoires de projets sont structurés par 2216 EPCI, dont 6 communautés urbaines (sur 16), 81 communautés d'agglomérations (sur 191) et 2129 communautés de communes (sur 2387). La règle de cohérence des périmètres des Pays avec ceux des EPCI est globalement bien respectée, puisque seuls 72 d'entre eux dérogent à la règle. Trois modèles de structuration intercommunale sont observés ${ }^{12}$ (tableau 2).

12. Auxquels s'ajoute une configuration de structuration intercommunale des Pays incomplète, mais qui est très minoritaire en 2010 et destinée à disparaître avec la couverture intégrale en EPCI. 
FAITS ET CHIFFRES

Tableau 2. Modèles de structuration intercommunale des Pays en 2010

\begin{tabular}{|c|c|c|c|c|c|c|c|c|c|c|}
\hline & \multicolumn{2}{|c|}{$\begin{array}{c}\text { Regroupement } \\
\text { com. com (1) }\end{array}$} & \multicolumn{2}{|c|}{$\begin{array}{l}\text { Regroupement } \\
\text { hiérarchisé (1) }\end{array}$} & \multicolumn{2}{|c|}{ Pays=Epci (1) } & \multicolumn{2}{|c|}{$\begin{array}{l}\text { Couverture } \\
\text { partielle (2) }\end{array}$} & \multicolumn{2}{|c|}{ Total } \\
\hline & $\begin{array}{l}\text { Ens } \\
\text { Pays }\end{array}$ & $\begin{array}{l}\text { dont } \\
\text { FPU }\end{array}$ & $\begin{array}{l}\text { Ens } \\
\text { Pays }\end{array}$ & $\begin{array}{l}\text { dont } \\
\text { FPU }\end{array}$ & $\begin{array}{l}\text { Ens } \\
\text { Pays }\end{array}$ & $\begin{array}{l}\text { dont } \\
\text { FPU }\end{array}$ & $\begin{array}{l}\text { Ens } \\
\text { Pays }\end{array}$ & $\begin{array}{l}\text { dont } \\
\text { FPU }\end{array}$ & Ens Pays & $\begin{array}{l}\text { dont } \\
\text { FPU }\end{array}$ \\
\hline \multirow{2}{*}{$\begin{array}{l}\text { Pays d'agglomé- } \\
\text { ration \& urbain }\end{array}$} & 64 & 30 & 73 & 49 & 10 & 7 & 7 & 3 & 154 & 89 \\
\hline & $42 \%$ & $34 \%$ & $47 \%$ & $55 \%$ & $6 \%$ & $8 \%$ & $5 \%$ & $3 \%$ & $100 \%$ & $100 \%$ \\
\hline \multirow{2}{*}{ Pays périurbains } & 51 & 25 & 1 & 1 & 6 & 2 & 4 & 3 & 62 & 31 \\
\hline & $82 \%$ & $81 \%$ & $2 \%$ & $3 \%$ & $10 \%$ & $6 \%$ & $6 \%$ & $10 \%$ & $100 \%$ & $100 \%$ \\
\hline \multirow{2}{*}{ Pays ruraux } & 33 & 14 & 0 & 0 & 5 & 1 & 1 & 1 & 39 & 16 \\
\hline & $85 \%$ & $88 \%$ & $0 \%$ & $0 \%$ & $13 \%$ & $6 \%$ & $3 \%$ & $6 \%$ & $100 \%$ & $100 \%$ \\
\hline \multirow{2}{*}{$\begin{array}{l}\text { Pays composites } \\
\text { non polarisés }\end{array}$} & 48 & 14 & 0 & 0 & 5 & 2 & 2 & 1 & 55 & 17 \\
\hline & $87 \%$ & $82 \%$ & $0 \%$ & $0 \%$ & $9 \%$ & $12 \%$ & $4 \%$ & $6 \%$ & $100 \%$ & $100 \%$ \\
\hline \multirow{2}{*}{$\begin{array}{l}\text { Pays composites } \\
\text { polarisés }\end{array}$} & 77 & 22 & 9 & 4 & 0 & 0 & 4 & 1 & 90 & 27 \\
\hline & $86 \%$ & $81 \%$ & $10 \%$ & $15 \%$ & $0 \%$ & $0 \%$ & $4 \%$ & $4 \%$ & $100 \%$ & $100 \%$ \\
\hline Ensemble des & 273 & 105 & 83 & 54 & 26 & 12 & 18 & 9 & 400 & 180 \\
\hline Pays & $68 \%$ & $58 \%$ & $21 \%$ & $30 \%$ & $7 \%$ & $7 \%$ & $5 \%$ & $5 \%$ & $100 \%$ & $100 \%$ \\
\hline
\end{tabular}

Source : DATAR 2010, ministère de I'Intérieur - DGCL2010.

Notes : (1) Lorsque $75 \%$ ou plus de la population du Pays appartiennent respectivement à au moins deux communautés de communes (type 1), à au moins une communauté d'agglomération et une communauté de communes (type 2), ou au maximum à un EPCI (type 3). (2) Lorsque moins de $75 \%$ de la population du Pays appartiennent à un EPCI.

- Les regroupements des communautés de communes de loin dominantes (7 Pays sur 10), qui s'observent dans tous les types spatiaux de Pays ; ils comptent en moyenne un peu plus de 5 EPCI par Pays. Un quart de ces Pays regroupent 2 ou 3 EPCI, $40 \%$ en regroupent entre 4 et 6 et $20 \%$ entre 7 et 9 . Les regroupements dominés par les communautés de communes à fiscalité professionnelle unique ${ }^{13}$ sont les plus représentés, mais dans 4 cas sur 10, le regroupement d'EPCI ne favorise pas la généralisation du régime de fiscalité unique dans les EPCI d'un même Pays. Les Pays à forte intégration fiscale relèvent davantage de facteurs régionaux (incitations des préfectures départementales, degré de coopération entre élus

13. Dans ce régime, l'EPCI se substitue progressivement aux communes pour la gestion et la perception, sur l'ensemble de son périmètre, du produit de la fiscalité professionnelle. Les regroupements sont dits dominés par le régime FPU lorsque $75 \%$ et plus de la population du Pays habitent dans un EPCI à FPU. locaux) qui apparaissent particulièrement favorables en Bretagne et dans le centre de la France, et au contraire moins marqués dans le nord-est (carte 2).

- Des regroupements de communautés urbaines ou d'agglomération et de communautés de communes (données dans le tableau 2 sous « regroupement hiérarchisé ») sont logiquement présents dans les Pays d'agglomération et les Pays composites ${ }^{14}$. Dans ces configurations, le nombre d'EPCI est un peu plus important que dans le groupe précédent, notamment dans les Pays composites. Les communautés de communes en FPU sont un peu plus fréquentes, mais l'association avec une intercommunalité urbaine obligatoirement en FPU ne semble pas favoriser significativement l'intégration fiscale dans ces autres EPCI.

14. On n'enregistre qu'un seul cas de regroupement de deux communautés d'agglomération dans le Pays de Belfort-Montbéliard. 
- Enfin, des Pays-communauté (données dans le tableau 2 sous Pays = Epci), très peu nombreux et présents majoritairement dans les Pays urbains. Cette configuration qui concerne des Pays de taille semblable aux Pays structurés par plusieurs EPCI, peut parfois résulter d'un choix volontariste local de constitution de grandes communautés de communes. La concentration de ces Pays dans les régions ChampagneArdenne, Poitou-Charentes et RhôneAlpes (carte 1) peut également résulter d'orientations préfectorales dans les départements et de stratégies régionales de structuration territoriale. Ce modèle d'organisation, que la réforme des collectivités territoriales de 2010 semble vouloir promouvoir, relève pour l'heure de l'exception.

Cet état des lieux de la structuration intercommunale actuelle des Pays montre que le modèle de regroupement d'EPCI est la norme tant en zone urbaine que rurale et que près de $80 \%$ d'entre eux regroupent au moins 5 EPCI. Si la taille des EPCI est très homogène en nombre de communes (14 en moyenne), leur dimension en population suit logiquement la densité de population, soit respectivement 7 800, 10200 et 13400 habitants pour les Pays ruraux, composites et périurbains. Enfin, le nombre d'EPCI par Pays varie sensiblement selon les types de Pays. Si $30 \%$ des Pays périurbains regroupent 3 EPCI ou moins, plus de 9 Pays composites sur 10 rassemblent au moins 4 EPCI.

\section{Une restructuration intercommunale pour quels territoires de projet ?}

\section{Des schémas départementaux de coopération intercommunale très hétérogènes}

Du fait de l'abrogation par la loi RCT de l'article 22 de la LOADDT relatif aux Pays, ces derniers sont rarement évoqués directement dans les SDCI adoptés en application de cette loi. Les Pays sont cependant impactés d'abord au travers de l'objectif d'élargissement significatif de
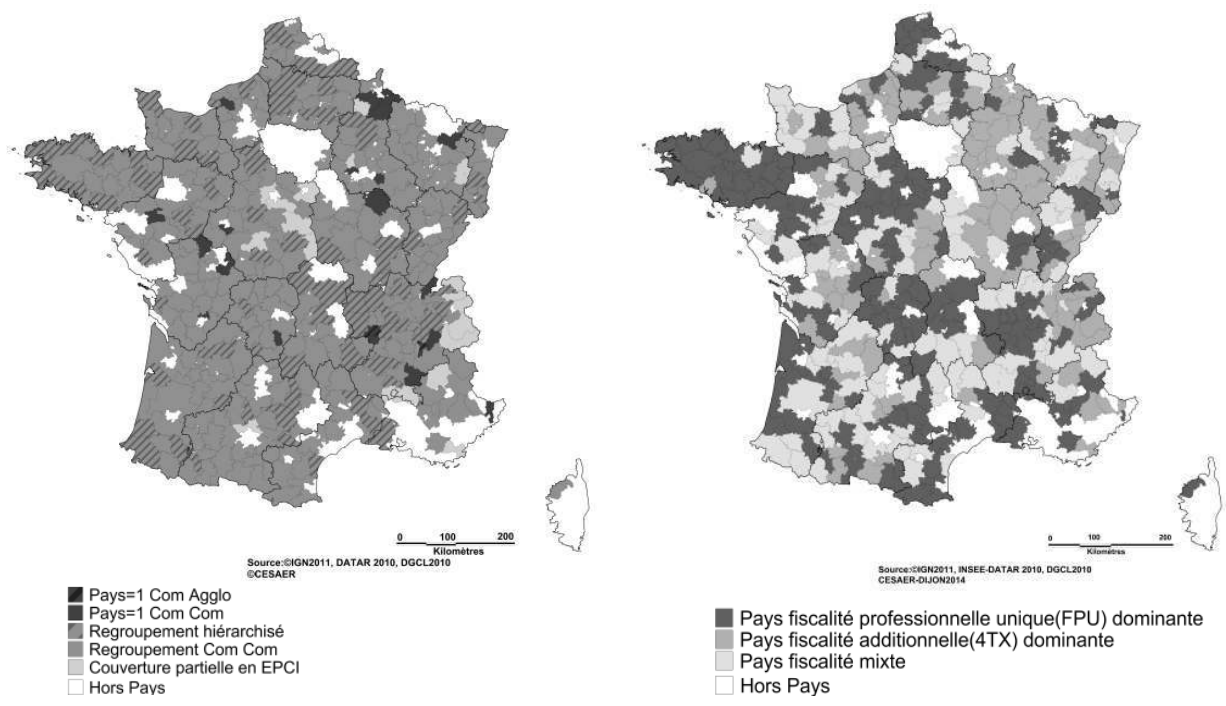
la taille des $\mathrm{EPCI}^{15}$. Ensuite, la réforme visait également la réduction significative du nombre de syndicats intercommunaux, ce qui aurait pu impacter les Pays dans la mesure où la moitié d'entre eux est structurée en syndicat mixte (le reste est essentiellement constitué en association). Par ailleurs, l'objectif de rationalisation des structures d'aménagement figurant également dans la loi s'est traduit par une attention portée aux Schémas de cohérence territoriale (SCoT).

L'examen de ces SDCI (encadré 2) fait apparaître une forte hétérogénéité, s'expliquant largement par le type de compromis passé entre le préfet et les principaux élus. Des propositions figurant dans les projets initiaux des préfets ne se retrouvent pas dans les versions finales adoptées, parfois sous l'effet d'amendements des élus des CDCI rejetant des propositions substantielles des préfets. Les SDCI se composent souvent de prescriptions d'application immédiate et de préconisations prospectives susceptibles d'être adoptées lors de la «clause de revoyure » désormais fixée à 2015. En effet, la recherche du consensus, lorsque des difficultés d'adhésion au projet sont apparues, a conduit à renvoyer à plus tard (avec ou sans précision de date) les aspects les plus délicats : ceux-ci sont mentionnés comme des orientations pour l'avenir ou des encouragements à une réflexion ultérieure.

\section{Une restructuration qui ne remet pas en cause l'échelle supra-intercommunale}

Sur les 63 SDCI étudiés, 23 SDCI mentionnent les Pays de diverses manières, certains schémas les évoquant au détour d'une situation particulière. La carte des

15. La population de 5000 habitants était posée comme un minimum par la loi, mais un objectif de 10000 habitants était la consigne donnée par le ministère de l'Intérieur aux préfets, il est aussi parfois (Eure) invoqué une règle des 30 (30 communes, 30000 habitants, 30000 hab.).
Pays ne figure que dans 13 SDCI, contrairement à celle des SCoT souvent présente et expressément prévue par la loi RCT contrairement aux Pays. Parfois, les Pays sont mentionnés au travers des syndicats mixtes, mais aussi plus largement comme territoires de coopération (rappelons que seulement un peu plus de la moitié sont organisés en syndicat mixte).

La prise en compte des Pays est donc extrêmement variable : du cas de l'Illeet-Vilaine qui appréhende son schéma à partir de la cartographie de ses six Pays, à la Seine-Maritime qui indique que «les Pays ne disposent pas de la personnalité juridique et s'appuient pour la plupart sur des syndicats mixtes qui comprennent des territoires plus vastes que les EPCI ». Ils sont parfois évoqués comme cadre de coopération établi permettant désormais d'aller plus loin dans l'intégration intercommunale par des fusions de communautés à leur échelle ou dans ce cadre. Dans d'autres cas, les Pays sont le cadre de réflexion et de référence des périmètres, ceci parfois en lien avec les SCoT. Il est cependant très rare de voir indiqué explicitement le maintien ou non de tel ou tel syndicat de Pays.

Les prescriptions des SDCI impactant négativement les Pays sont dans les versions finales adoptées par les Commissions départementales de coopération intercommunale $(\mathrm{CDCI})$, beaucoup moins nombreuses que ne pouvaient le laisser annoncer les projets présentés par les préfets. Par exemple, dans le département de la Vienne, les propositions du préfet conduisaient à l'origine à la disparition de fait de la plupart des Pays du département, ce qui n'est plus le cas dans la version votée par la CDCI.

Dans certains cas, la restructuration des communautés peut se traduire par un franchissement du périmètre du Pays du fait du rattachement de communes appartenant à deux Pays différents, ou de la fusion de 
communautés adhérant jusque-là à deux Pays distincts. Alors que la loi RCT a mis fin à la procédure de redéfinition du périmètre des Pays, nous avons relevé qu'un SDCI (Maine et Loire) traite de cette problématique. Enfin, les questions institutionnelles (présidence, compétences), fiscales et financières ont généralement été au centre des préoccupations des élus, au détriment de celles d'aménagement et de développement des territoires.

En première analyse, trois grands cas de figure méritent une attention particulière dans les SDCI :

- Les fusions de communautés en une seule, notamment dans les petits Pays, avec l'intérêt de coopérations à une échelle plus large (cf. infra).

- Les reconfigurations de Pays aux marges du fait de reconfiguration de communautés (fusion, rattachement de communes isolées), avec la nécessité de revoir obligatoirement la liste des membres du Pays en cas de syndicat mixte.

- Les risques d'implosion de Pays du fait de déséquilibres internes résultant de la constitution d'une grosse communauté de communes (ou d'agglomération) au côté d'une ou deux petites : ici, des observations seront à mener pour voir comment évoluent ces Pays (exemples : Gâtine dans les Deux-Sèvres, Avre et Iton dans l'Eure, 7 Vallées dans le Pas-de-Calais).

Au total, sur la base des 63 SDCI étudiés, on enregistre une diminution de $21 \%$ du nombre d'EPCI, faisant passer le nombre moyen d'EPCI par Pays de 5,6 avant les SDCI à 4,4 après (tableau 3), sachant que cette structuration intègre, outre les fusions d'EPCI, l'extension des intercommunalités aux communes isolées. Si ces évolutions touchent tous les types de Pays, l'intensité des restructurations est d'autant plus forte que le nombre d'EPCI était élevé avant la réforme. Les impacts des structurations sont donc marqués pour les Pays urbains et d'agglomération, les Pays composites ainsi que les Pays ruraux maillés, alors qu'elle est plus limitée pour les Pays périurbains. Ce sont les Pays mono-communautaires et ceux regroupant deux EPCI qui augmentent le plus en nombre à l'issue des SDCI. Cette évolution, qui s'observe pour l'essentiel dans les Pays urbains et composites, atteste dans les deux cas d'une capacité des collectivités locales à dépasser les oppositions urbain/ rural pour construire des intercommunalités à l'échelle de territoire plus hétérogène au plan spatial. On observe ensuite une diminution systématique du nombre

Tableau 3. Évolution de la composition intercommunale selon la structure spatiale des Pays (sur la base des 294 Pays concernés par les 63 SDCl étudiés)

\begin{tabular}{|c|c|c|c|c|}
\hline \multirow{2}{*}{ Typologie spatiale des Pays } & \multirow{2}{*}{$\begin{array}{l}\text { Nombre } \\
\text { de Pays }\end{array}$} & \multicolumn{2}{|c|}{ Nombre d'EPCI/Pays } & \multirow{2}{*}{$\begin{array}{c}\text { Évolution } \\
\text { Avant/après } \\
\text { en \% }\end{array}$} \\
\hline & & Avant SDCl & Après SDCl & \\
\hline Pays d'agglomération et urbains & 124 & 5,9 & 4,6 & -22 \\
\hline dont Pays d'agglomération & 48 & 6,9 & 5,4 & -22 \\
\hline dont Pays urbains & 76 & 5,3 & 4,2 & -21 \\
\hline Pays périurbains & 49 & 3,6 & 3,1 & -14 \\
\hline Pays ruraux & 23 & 4,2 & 3,3 & -21 \\
\hline dont Pays ruraux maillés & 10 & 6,1 & 4,4 & -28 \\
\hline Pays composites & 98 & 6,4 & 4,9 & -23 \\
\hline Dont Pays composites monopolaires & 25 & 6,5 & 4,5 & -31 \\
\hline Ensemble Pays & 294 & 5,6 & 4,4 & -21 \\
\hline
\end{tabular}

Source : DATAR et auteurs d'après SDCI. 
de Pays composés de plus de huit EPCI et une disparition des configurations de Pays éclatées en un grand nombre d'EPCI (le nombre maximum d'EPCI dans un Pays passe de 20 à 14).

\section{Des modes de restructurations différenciés dans l'espace}

Nous avons centré notre analyse sur deux types de structuration intercommunale particulièrement impactés par la réforme de la carte des EPCI (APFP, 2012) ${ }^{16}$ :

- Les Pays à faible nombre de communautés (entre une et trois), dans la mesure où le Pays est généralement justifié comme moyen de faire coopérer des EPCI à fiscalité propre et où l'esprit de la loi RCT était de transformer les Pays en une seule communauté.

- Les Pays d'agglomération, qui avaient pour objectif implicite de faire coopérer espaces urbain et périurbain selon la loi Voynet, et d'être une instance d'apprentissage avant d'aller vers une intégration plus poussée dans une communauté d'agglomération étendue.

\section{Le devenir des Pays au faible nombre de communautés}

Sur les 63 schémas, avant adoption des SDCI, concernant 293 Pays, étaient dénombrés 25 Pays constitués d'un seul EPCI, 22 composés de deux EPCI, 51 incluant 3 EPCI. Les évolutions qui ressortent des SDCI sont les suivantes :

- Des Pays à une communauté plus nombreux, mais qui restent marginaux (12\% des Pays étudiés)

Les Pays composés d'une seule communauté et dont le périmètre se superpose ont vocation à disparaître selon la loi RCT. Sont concernés 25 Pays dans les schémas

16. APFP (2012). Impacts des SDCI sur les Pays. Analyse résumée par département. http://www. Pays.asso.fr/spip.php?article1380, 23 p. étudiés. Parfois, ce sont des communautés résultant de la fusion récente des anciennes communautés constituant le Pays (Pays urbain de Caux-Vallée Seine ou de Grésivaudan). Certains sont également des Pays qui n'avaient toujours pas été reconnus au titre de la LOADDT : Maures, Entre Touvre et Charente. Au total, 36 Pays doivent passer à une communauté : 13 Pays composites, 9 Pays périurbains, 9 Pays urbains, 3 Pays d'agglomération, 3 Pays ruraux, et un Pays (Vésubie) est intégré dans la première Métropole créée (Nice).

- Les Pays à deux communautés : peu de fusions à une communauté

Sur 22 Pays concernés dans les SDCI étudiés, quatre prescriptions de fusions de communautés à l'échelle du Pays ont été faites. Au total, le nombre de Pays devant passer à deux communautés atteint 37, et ceci concerne 11 Pays urbains, 9 Pays composites, 8 Pays périurbains, 5 Pays d'agglomération et 4 Pays ruraux. Des fusions proposées, bien que non retenues, ont été transformées en objectif de renforcement des coopérations intercommunales, de rapprochement, de fusions à prévoir, ou en recommandations en termes de périmètres des syndicats mixtes de Pays (extension ou réduction).

- Les Pays composés de 3 EPCI : des évolutions concernant tous les types de Pays

Sont concernés 51 Pays dans les SDCI étudiés. Pour cinq Pays, le SDCI propose le regroupement des trois EPCI pour en constituer un seul (ceux-ci ont alors vocation, s'ils sont syndicat mixte, à disparaître en tant que tels). Pour dix autres Pays, les EPCI passeraient de 3 à 2 et ils n'auraient donc a priori aucune raison de disparaître. Pour plusieurs autres, le SDCI propose, à terme, des rapprochements ou une fusion à l'échelle du Pays à une échéance fixée ou par étapes. Au total, le nombre de Pays passant à 3 EPCI est de 53, et ceci concerne l'ensemble des catégories de 
Pays. Enfin, les restructurations plus importantes sont plus rares. On peut noter deux cas de fusion de 4 communautés en 1 Pays, 3 cas de fusion de 5 communautés, et un cas de fusion de 6 communautés : le Gard Rhodanien (47 communes). Le passage de Pays à une communauté à 2 communautés (Maures) ou à 5 (Loire Beauce) s'explique par la couverture intégrale du Pays en EPCI.

\section{L'évolution des Pays d'agglomération}

Dans l'esprit de la loi Voynet, les Pays construits autour d'une agglomération étaient conçus pour faire coopérer espaces urbain et périurbain, et comme une instance d'apprentissage avant d'aller vers une intégration plus poussée dans une communauté d'agglomération étendue à une échelle se rapprochant de l'aire urbaine (selon certains textes de la DATAR, 2011) ${ }^{17}$. Or, en 2004, seulement $9 \%$ des aires urbaines vivaient sous le régime politique d'une seule intercommunalité englobant la quasi-totalité de l'aire urbaine (Estèbe, 2008) ${ }^{18}$.

La totalité des Pays d'agglomération au sens de la typologie ici utilisée (62) comprend une communauté d'agglomération ou urbaine (sauf trois), et dans un cas deux communautés (Belfort-Montbéliard), alors qu'au sein de l'ensemble des 92 Pays urbains, seulement 17 Pays comportent une communauté d'agglomération.

Nous avons analysé les évolutions prescrites pour 38 Pays d'agglomération sur la base des SDCI arrêtés. Il en ressort des évolutions très modérées : 13 Pays connaissent le statu quo, 14 voient la disparition d'une communauté, généralement par intégration d'une communauté périurbaine dans la communauté d'agglomération. Les cas

17. DATAR (2011). Contrats d'agglomération, mode d'emploi, septembre. www.datar.gouv.fr/ sites/default/files/datar/contrat-agglos.pdf.

18. Estèbe P. (2008). Gouverner la ville mobile. Intercommunalité et démocratie locale. Paris, PUF, coll. « La ville en débat », 76 p. le plus ambitieux se situent dans le Pays Rémois (8 communautés sur 17), dans le Pays de Sambre Avesnois, qui voit la disparition de 7 communautés et dans le Pays de Châlons-en-Champagne (moins 6 communautés sur 11).

Dans certains cas, le maintien du statu quo est justifié par des pratiques de coopération récentes au niveau des communautés périphériques (cf. Pays de Limoges) ou par souci d'équilibre : ainsi au Mans, l'enjeu selon le SDCI est de «conforter un schéma intercommunal "polycentrique sur l'aire métropolitaine", ce qui consiste à stabiliser peu ou prou Le Mans Métropole dans un périmètre correspondant à son "unité urbaine”, c'est-à-dire la zone urbaine "dense", à conforter les communautés de communes périphériques de taille importante [...], et à faire jouer pleinement au futur SCoT, à l'échelle de l'ensemble de l'aire métropolitaine, son rôle de mise en cohérence et de régulation de ces territoires ». Cette référence aux SCoT peut s'avérer inadaptée, notamment dès lors que certains SCoT peuvent être conçus à une échelle infra-Pays (par exemple en Pas de Calais, Pays Boulonnais comprenant deux SCoT). La référence à l'aire urbaine est souvent jugée trop vaste, référence au demeurant non retenue par la loi RCT. Une instance de type Pays devrait donc rester utile pour porter les coopérations à une échelle supra-communautaire, y compris dans ces espaces urbains. Il est à noter d'ailleurs à l'échelle de Pays d'agglomération, des projets de "pôles métropolitains » tels que définis par la loi RCT du 16 décembre 2010. Sur les six Pôles créés début 2012, un concerne directement un Pays établi sur le même périmètre (Brest).

$$
\begin{gathered}
* \\
* *
\end{gathered}
$$

Deux voies de structuration intercommunale des territoires se sont longtemps tourné le dos, celle de l'intercommunalité promue par le ministère de l'Intérieur et les 
conseils généraux et celle des territoires de projets portée par la DATAR et par les conseils régionaux. Les années 2000 ont marqué le rapprochement de ces deux démarches et leur mise en cohérence progressive qui ont permis une généralisation encore jamais atteinte de ce double niveau de structuration. Il n'est pas surprenant, dans un contexte de tension sur la dépense publique, que se pose aujourd'hui la question de la pertinence et de l'efficacité de cette organisation.

La réforme des collectivités territoriales souhaite amener à plus d'intégration entre échelle intercommunale de gestion et échelle intercommunale de projet de territoire. Le constat tiré de l'analyse des SDCI est que la restructuration de l'intercommunalité sera significative et devrait sans aucun doute accroître les capacités de travail stratégique et d'intervention des EPCI, mais il semble peu probable que cette restructuration remette en cause la légitimité de la distinction des deux niveaux d'action. On peut au contraire faire l'hypothèse que la restructuration de l'intercommunalité peut conduire à renforcer le modèle de coopération de la construction des Pays et d'en faire évoluer les périmètres et les missions afin d'accroître leur capacité à se saisir des enjeux de développement économique et de l'équité territoriale en matière d'accès aux équipements, des services publics et du logement, de l'alimentation de proximité, de la gestion de l'espace et des ressources naturelles. L'adoption en 2013, dans le cadre de la loi de modernisation de l'action publique territoriale et d'affirmation des métropoles, d'un article portant sur la création de pôles d'équilibre territorial et rural vise notamment à donner un nouveau souffle à ces formules de coopération de type Pays. Dans ce contexte, les configurations territoriales de "pôles » structurant les aires urbaines, les espaces ruraux les plus éloignés des villes et les espaces intermédiaires que révèlent les Pays composites devraient être de nature à faciliter l'adaptation des politiques publiques aux enjeux spécifiques de développement en s'appuyant sur un maillage de collectivités intercommunales solides. 


\section{ANNEXE}

Méthodologie de typologie spatiale des Pays

\begin{tabular}{|c|c|c|}
\hline \multicolumn{2}{|c|}{ Typologie spatiale des Pays } & \multirow{2}{*}{ Critères de construction des types } \\
\hline Types agrégés & Types détaillés & \\
\hline \multirow{2}{*}{$\begin{array}{l}\text { Pays } \\
\text { d'Agglomération } \\
\text { et urbains }\end{array}$} & $\begin{array}{l}\text { Pays } \\
\text { d'agglomération }\end{array}$ & $\begin{array}{l}\text { - \% population résidant dans grande ou moyenne aire urbaine } \geq 70 \\
\text { - grand pôle urbain + moyen pôle urbain } \geq 1 \\
\text { - Pôle urbain de plus de } 50000 \text { habitants }\end{array}$ \\
\hline & Pays urbains & $\begin{array}{l}\text { - \% population résidant dans grande ou moyenne aire urbaine } \geq 70 \\
\text { - grand pôle urbain + moyen pôle urbain } \geq 1 \\
\text { - } 1 \text { pôle urbain de moins de } 50000 \text { habitants }\end{array}$ \\
\hline \multirow[b]{2}{*}{ Pays périurbains } & $\begin{array}{l}\text { Pays périurbains } \\
\text { sans relais }\end{array}$ & $\begin{array}{l}\text { - \% population résidant en communes périurbaines des grands ou } \\
\text { moyens pôles et autres communes périurbaines multipolaires } \geq 70 \\
\text { - Aucun pôle }\end{array}$ \\
\hline & $\begin{array}{l}\text { Pays périurbains } \\
\text { avec relais }\end{array}$ & $\begin{array}{l}\text { - \% population résidant en communes périurbaines des grands ou } \\
\text { moyens pôles et autres communes périurbaines multipolaires } \geq 70 \\
\text { - Aucun grand et moyen pôle (respectivement }>10000 \text { et } 5000 \text { à } \\
10000 \text { emplois) } \\
\text { - Au moins un petit pôle (de } 1500 \text { à } 5000 \text { emplois) }\end{array}$ \\
\hline \multirow{3}{*}{ Pays ruraux } & Pays ruraux & $\begin{array}{l}\text { - \% population résidant dans communes isolées hors de l'influence } \\
\text { des grands et moyens pôles ou dans aire urbaine de petit pôle } \geq 70 \\
\text { - Aucun pôle }\end{array}$ \\
\hline & $\begin{array}{l}\text { Pays ruraux de } \\
\text { petites villes }\end{array}$ & $\begin{array}{l}\text { - \% population résidant dans communes isolées hors de l'influence } \\
\text { des grands et moyens pôles ou dans aire urbaine de petit pôle } \geq 70 \\
\text { - } 1 \text { petit pôle }\end{array}$ \\
\hline & $\begin{array}{l}\text { Pays ruraux } \\
\text { maillés }\end{array}$ & $\begin{array}{l}\text { - \% population résidant dans communes isolées hors de l'influence } \\
\text { des grands et moyens pôles ou dans aire urbaine de petit pôle } \geq 70 \\
\text { - } 2 \text { petits pôles ou plus }\end{array}$ \\
\hline \multirow{3}{*}{ Pays composites } & \begin{tabular}{|l} 
Pays composites \\
non polarisés
\end{tabular} & $\begin{array}{l}\text { - Non-Pays d'agglomération, périurbains, ruraux } \\
\text { - Aucun pôle }\end{array}$ \\
\hline & $\begin{array}{l}\text { Pays composites } \\
\text { monopolaires }\end{array}$ & $\begin{array}{l}\text { - Non-Pays d'agglomération, périurbains, ruraux } \\
\text { - } 1 \text { grand ou moyen pôle }\end{array}$ \\
\hline & $\begin{array}{l}\text { Pays composites } \\
\text { multipolaires }\end{array}$ & $\begin{array}{l}\text { - Non-Pays d'agglomération, périurbains, ruraux } \\
\text { - } 2 \text { grands, moyens ou petits pôles ou plus }\end{array}$ \\
\hline
\end{tabular}

